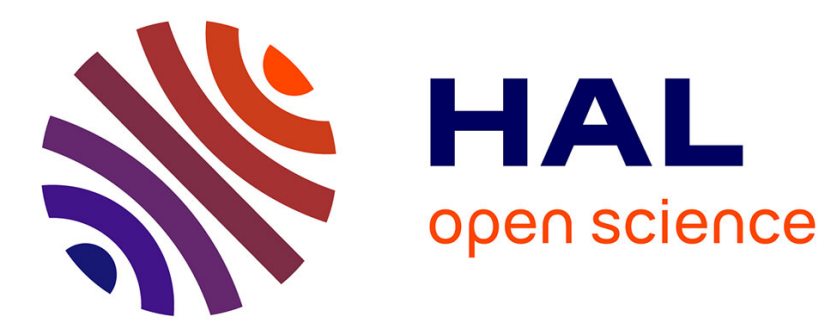

\title{
On homomorphisms between products of median algebras
}

\author{
Miguel Couceiro, Stephan Foldes, Gerasimos C. Meletiou
}

\section{To cite this version:}

Miguel Couceiro, Stephan Foldes, Gerasimos C. Meletiou. On homomorphisms between products of median algebras. Algebra Universalis, 2017, 78 (4), pp.545-553. 10.1007/s00012-017-0468-6 . hal01500223

\section{HAL Id: hal-01500223 \\ https://hal.inria.fr/hal-01500223}

Submitted on 2 Apr 2017

HAL is a multi-disciplinary open access archive for the deposit and dissemination of scientific research documents, whether they are published or not. The documents may come from teaching and research institutions in France or abroad, or from public or private research centers.
L'archive ouverte pluridisciplinaire HAL, est destinée au dépôt et à la diffusion de documents scientifiques de niveau recherche, publiés ou non, émanant des établissements d'enseignement et de recherche français ou étrangers, des laboratoires publics ou privés. 


\title{
ON HOMOMORPHISMS BETWEEN PRODUCTS OF MEDIAN ALGEBRAS
}

\author{
MIGUEL COUCEIRO, STEPHAN FOLDES, AND GERASIMOS C. MELETIOU
}

\begin{abstract}
Homorphisms of products of median algebras are studied with particular attention to the case when the codomain is a tree. In particular, we show that all mappings from a product $\mathbf{A}_{1} \times \cdots \times \mathbf{A}_{n}$ of median algebras to a median algebra $\mathbf{B}$ are essentially unary whenever the codomain $\mathbf{B}$ is a tree. In view of this result, we also characterize trees as median algebras and semilattices by relaxing the defining conditions of conservative median algebras.
\end{abstract}

\section{INTRODUCTION}

Median algebras have been investigated by several authors in the context of distributive algebras, semilattices, graphs and hypergraphs and in the framework of convex and metric spaces (see, e.g., $[1,2,4,5,10,14]$ ). A median algebra is a structure $\mathbf{A}=\langle A, \mathbf{m}\rangle$ for a set $A$ and a ternary symmetric operation $\mathbf{m}: A^{3} \rightarrow A$, called median, such that

$$
\begin{gathered}
\mathbf{m}(x, x, y)=x, \\
\mathbf{m}(\mathbf{m}(x, y, z), t, u)=\mathbf{m}(x, \mathbf{m}(y, t, u), \mathbf{m}(z, t, u)) .
\end{gathered}
$$

In fact, axiom systems using only 4 variables are also known $[2,12]$.

It is well known [14] that each element $a$ of a median algebra $\mathbf{A}$ gives rise to a median semilattice $\left\langle A, \leqslant_{a}\right\rangle$ where $\leqslant_{a}$ is given by

$$
x \leqslant_{a} y \quad \Longleftrightarrow \quad \mathbf{m}(a, x, y)=x .
$$

In this semilattice $a$ is the bottom element and the associated binary operation, denoted $\wedge_{a}$, is defined by $x \wedge_{a} y=\mathbf{m}(a, x, y)$. As shown in [14], median semilattices coincide exactly with those $\wedge$-semilattices whose principal ideals

$$
\downarrow x:=\{y \in A: y \leqslant a x
$$

are distributive lattices, and such that for any $a, b, c \in A, a \wedge b, b \wedge c, c \wedge a$ have a supremum whenever each pair of these meets is bounded above. In such a case, we can define a median operation by

$$
\mathbf{m}_{\leqslant}(x, y, z)=(x \wedge y) \vee(x \wedge z) \vee(z \wedge y),
$$

for every $x, y, z \in A$. Moreover, $\mathbf{m}=\mathbf{m}_{\leqslant_{a}}$ for every median algebra $\mathbf{A}=\langle A, \mathbf{m}\rangle$, and every $a \in A[1]$. Similarly, every distributive lattice gives rise to a median algebra using (1.1) and the converse also holds whenever there are $a, b \in A$ such that $\mathbf{m}(a, x, b)=x$ for every $x \in A$.

Another noteworthy connection is to median graphs, i.e., connected graphs having the property that for any three vertices $a, b, c$, there is exactly one vertex $x$ which lies on a shortest path between each pair of vertices in $\{a, b, c\}$. As shown in [1], the covering graph (i.e., undirected Hasse diagram) of every median semilattice whose intervals are finite is a median graph. Conversely, every median graph is the covering graph of a median semilattice. For further background see, e.g., [2].

Date: January 2, 2017, 17:15. 
In [8] the authors studied conservative median algebras, i.e., median algebras that satisfy $\mathbf{m}(x, y, z) \in\{x, y, z\}$, for all $x, y, z \in A$. Apart from the 4-element Boolean algebra, it was shown that conservative median algebras can be represented by two lower bounded chains whose least elements are identified (thus, they can always be regarded as chains). The authors in [8] also provided complete descriptions of median-homomorphisms between conservative median algebras (with at least 5 elements). We recall the main results of [8] in the two following theorems.

Theorem 1.1 ([8]). Let $\mathbf{A}$ and $\mathbf{B}$ be two conservative median algebras (thought of as chains) with at least five elements. A map $f: \mathbf{A} \rightarrow \mathbf{B}$ is a median homomorphism if and only if $f$ is monotone (i.e., order-preserving or order-reversing).

This result was then lifted, by making use of dual topological spaces of median algebras, to median-homomorphisms between products of conservative median algebras. For each positive integer $n$, we set $[n]=\{1, \ldots, n\}$.

Theorem 1.2 ([8]). Let $\mathbf{A}=\mathbf{C}_{1} \times \cdots \times \mathbf{C}_{n}$ and $\mathbf{B}=\mathbf{D}_{1} \times \cdots \times \mathbf{D}_{k}$ be two finite products of conservative median algebras (thought of as chains). Then $f: \mathbf{A} \rightarrow \mathbf{B}$ is a median homomorphism if and only if there exist $\sigma:[k] \rightarrow[n]$ and monotone maps $f_{i}: \mathbf{C}_{\sigma(i)} \rightarrow \mathbf{D}_{i}$ for $i \in[k]$, such that $f=\left(f_{\sigma(1)}, \ldots, f_{\sigma(k)}\right)$.

In the particular case when $k=1$,

$$
f: \mathbf{C}_{1} \times \cdots \times \mathbf{C}_{n} \rightarrow \mathbf{D}
$$

is a median homomorphism if and only if there is $j \in[n]$ and a monotone map $g: \mathbf{C}_{j} \rightarrow \mathbf{D}$ such that $f=g \circ \pi_{j}$.

In this paper we generalize in particular these results from [8]. Instead of conservative median algebras (thought of as chains) we deal with trees (i.e., acyclic undirected graphs) when thought of as semilattices: a $\wedge$-semilattice (resp. $\vee$ semilattice) is said to be a tree if no pair of incomparable elements have an upper (resp. lower) bound. As we will see, such median algebras are obtained by relaxing the condition of being conservative: we say that a median algebra $\mathbf{A}$ is a (2:3)-median semilattice if $\mathbf{m}(x, y, z) \in\{x \wedge y, y \wedge z, z \wedge x\}$ for all $x, y, z \in A$.

\section{Preliminary Results}

As discussed in Section 1, when there is no danger of ambiguity, we will not distinguish between median algebras and median semilattices. They will be denoted by bold roman capital letters $\mathbf{A}, \mathbf{B}, \ldots$, while their universes will be denoted by italic roman capital letters $A, B, \ldots$. We will assume that universes of structures are nonempty, possibly infinite, sets.

Let $\mathbf{A}=\langle A, \mathbf{m}\rangle$ be a median algebra and let $a, b \in A$. The convex hull of $\{a, b\}$ or the interval from $a$ to $b$, denoted by $[a, b]$, is defined by

$$
[a, b]:=\{t \in A: t=\mathbf{m}(a, t, b)\}=\{\mathbf{m}(a, t, b): t \in A\} .
$$

Proposition 2.1 ([5]). Let $\mathbf{A}=\langle A, \mathbf{m}\rangle$ be a median algebra, and consider $a, b, c \in$ A. Then

$$
[a, b] \cap[b, c] \cap[a, c]=\{\mathbf{m}(a, b, c)\} .
$$

Every interval $[a, b]$ in a median algebra can be endowed with a distributive lattice structure. To this purpose, for $a \in A$ recall the binary operation $\wedge_{a}: A^{2} \rightarrow A$ defined by:

$$
x \wedge_{a} y:=\mathbf{m}(a, x, y), \quad \text { for }(x, y) \in A^{2} .
$$

It is easy to verify that $\left\langle[a, b], \wedge_{a}, \wedge_{b}\right\rangle$ is a distributive lattice with $a$ and $b$ as the least and greatest elements, respectively.

Moreover, we also have a general description of median-preserving mappings between two median algebras. 
Theorem 2.2. Let $\mathbf{A}$ and $\mathbf{B}$ be median algebras, and consider a mapping $f: A \rightarrow$ $B$. Then the following assertions are equivalent.

(i) $f$ is a median-homomorphism.

(ii) For all $p \in A, f:\left\langle A, \wedge_{p}\right\rangle \rightarrow\left\langle B, \wedge_{f(p)}\right\rangle$ is a semilattice-homomorphism.

(iii) For all $p \in A, f:\left\langle A, \leqslant_{p}\right\rangle \rightarrow\left\langle B, \leqslant_{f(p)}\right\rangle$ is an order-homomorphism.

(iv) For all $a, b \in A, f([a, b]) \subseteq[f(a), f(b)]$.

Proof. $(i) \Longrightarrow($ ii $)$ : Suppose that $f$ is a median-homomorphism and $p \in A$. Then

$$
f\left(a \wedge_{p} b\right)=f(\mathbf{m}(p, a, b))=\mathbf{m}(f(p), f(a), f(b))=f(a) \wedge_{f(p)} f(b) .
$$

(ii) $\Longrightarrow($ iii $)$ : Straightforward.

$($ iii $) \Longrightarrow($ iv $)$ : Let $t \in[a, b]$. Then $a \leqslant_{a} t \leqslant_{a} b$, and thus $f(a) \leqslant_{f(a)} f(t) \leqslant_{f(a)}$ $f(b)$ from which it follows that $f(t) \in[f(a), f(b)]$. In other words, $f([a, b]) \subseteq$ $[f(a), f(b)]$.

$(i v) \Longrightarrow(i)$ : Let $a, b, c \in A$. By Proposition 2.1, we have

$$
\{\mathbf{m}(a, b, c)\}=[a, b] \cap[b, c] \cap[c, a] .
$$

By $(i v)$, we then conclude that

$$
f(\mathbf{m}(a, b, c)) \in[f(a), f(b)] \cap[f(b), f(c)] \cap[f(c), f(a)]=\{\mathbf{m}(f(a), f(b), f(c))\} .
$$

Therefore, $f(\mathbf{m}(a, b, c))=\mathbf{m}(f(a), f(b), f(c))$.

Corollary 2.3. Let $f: A \rightarrow B$ be a median homomorphism between two median algebras $\mathbf{A}$ and $\mathbf{B}$. Then, for every $a, b \in A, f$ is also a lattice homomorphism from $\left\langle[a, b], \wedge_{a}, \wedge_{b}\right\rangle$ to $\left\langle[f(a), f(b)], \wedge_{f(a)}, \wedge_{f(b)}\right\rangle$.

\section{A RElaxation of CONSERVATiveness: TREes AS MEDiAn AlgEBras}

In this section we focus on median algebras that are trees. In the particular case of chains, it was shown that these median algebras are exactly those that are conservative, i.e.,

$$
\mathbf{m}(x, y, z) \in\{x, y, z\}, \quad x, y, z \in A .
$$

To characterise median semilattices that are trees, we propose the following relaxation of conservativeness. We say that a median algebra, thought of as a median semilattice $\mathbf{A}=\langle A, \wedge\rangle$, is a (2:3)-median semilattice if for every $x, y, z \in$ $A$, we have

$$
\mathbf{m}(x, y, z):=(x \wedge y) \vee(x \wedge z) \vee(z \wedge y) \in\{x \wedge y, y \wedge z, z \wedge x\} .
$$

We start with a simple yet useful observation.

Remark 3.1. Let $\mathbf{A}=\langle A, \mathbf{m}\rangle$ be a median algebra such that $\left\langle A, \leqslant_{c}\right\rangle$ is a tree for some (or, equivalently, all) $c \in A$. Then, for all $a, b \in A$, the convex hull $[a, b]$ is a chain from $a$ to $b$.

Theorem 3.2. Let $\mathbf{A}=\langle A, \mathbf{m}\rangle$ be a median algebra. Then the following assertions are equivalent.

(i) There is $p \in A$ such that $\mathbf{A}=\left\langle A, \leqslant_{p}\right\rangle$ is a (2:3)-median semilattice.

(ii) For all $p \in A, \mathbf{A}=\left\langle A, \leqslant_{p}\right\rangle$ is a (2:3)-median semilattice.

(iii) There is $p \in A$ such that $\mathbf{A}=\left\langle A, \leqslant_{p}\right\rangle$ is a tree.

(iv) For all $p \in A, \mathbf{A}=\left\langle A, \leqslant_{p}\right\rangle$ is a tree.

(v) For all $a, b \in A$, the bounded distributive lattice $\left\langle[a, b], \wedge_{a}, \wedge_{b}\right\rangle$ is a chain.

Proof. $($ ii $) \Longrightarrow(i)$ : Straightforward.

$(i) \Longrightarrow$ (iii): Suppose that $\mathbf{A}=\left\langle A, \leqslant_{p}\right\rangle$ is a $(2: 3)$-median semilattice. To show that it is a tree it suffices to show that for every pair $a, b \in A$ with a common upper bound, we have $a \leqslant_{p} b$ or $b \leqslant_{p} a$. 
So suppose that $c \in A$ is a common upper bound of $a$ and $b$, that is, $\mathbf{m}(p, a, c)=a$ and $\mathbf{m}(p, b, c)=b$. Since $\mathbf{A}=\left\langle A, \leqslant_{p}\right\rangle$ is a $(2: 3)$-median semilattice and $c$ is an upper bound of $a$ and $b$,

$$
\mathbf{m}(c, a, b) \in\{\mathbf{m}(p, a, b), a, b\} .
$$

Note that since $\mathbf{m}$ is a median,

$$
\mathbf{m}(p, \mathbf{m}(a, c, b), a)=\mathbf{m}(b, \mathbf{m}(a, a, p), \mathbf{m}(c, a, p))=\mathbf{m}(b, a, a)=a,
$$

and thus $a \leqslant_{p} \mathbf{m}(c, a, b)$. Similarly, we have $b \leqslant_{p} \mathbf{m}(c, a, b)$.

Now, if $\mathbf{m}(c, a, b)=\mathbf{m}(p, a, b)$ in $(3.1)$, then $\mathbf{m}(c, a, b) \leqslant_{p} a$ since $\mathbf{m}(p, a, b) \leqslant_{p} a$. Hence, $a=\mathbf{m}(c, a, b)$. Similarly, we also have $b=\mathbf{m}(c, a, b)$, and thus $a=b$.

If $\mathbf{m}(c, a, b)=a$ in $(3.1)$, then

$$
\mathbf{m}(p, a, b)=\mathbf{m}(\mathbf{m}(c, a, b), p, b)=\mathbf{m}(a, \mathbf{m}(c, p, b), \mathbf{m}(b, p, b))=\mathbf{m}(a, b, b)=b,
$$

and thus $b \leqslant_{p} a$. Similarly, if $\mathbf{m}(c, a, b)=b$ in (3.1), we conclude that $a \leqslant_{p} b$.

Since in all possible cases we have that $a \leqslant_{p} b$ or $b \leqslant_{p} a, \mathbf{A}=\left\langle A, \leqslant_{p}\right\rangle$ is a tree.

$($ iii $) \Longrightarrow($ iv $)$ : Straightforward.

$(i v) \Longrightarrow(v)$ : Let $s, t \in[a, b]$. Thus $a \leqslant_{a} s, t \leqslant_{a} b$, and $s, t$ have a common upper bound in $\left\langle A, \leqslant_{a}\right\rangle$. As $\left\langle A, \leqslant_{a}\right\rangle$ is a tree, $s$ and $t$ cannot be incomparable, and hence the interval $[a, b]$ is a chain.

$(v) \Longrightarrow(i v)$ : Let $a, b, p \in A$ and suppose that $a$ and $b$ are incomparable in $\mathbf{A}=\left\langle A, \leqslant_{p}\right\rangle$. Note that $p \leqslant_{p} a, b$.

To obtain a contradiction, suppose that $a$ and $b$ have a common upper bound $d \in A$, that is, $p \leqslant_{p} a, b \leqslant_{p} d$. However, by $(v)$ the interval $[p, d]$ is a chain and thus we must have $a \leqslant_{p} b$ or $b \leqslant_{p} a$, which constitutes the desired contradiction.

$(i v) \Longrightarrow(i i)$ : Let $p \in A$. As $\mathbf{A}=\left\langle A, \leqslant_{p}\right\rangle$ is a tree, for every $a, b, c \in A$, we have that $a \wedge b, b \wedge c$ and $c \wedge a$ are pairwise comparable, and thus

$$
\mathbf{m}(a, b, c) \in\{a \wedge b, b \wedge c, c \wedge a\} .
$$

Hence, $\mathbf{A}=\left\langle A, \leqslant_{p}\right\rangle$ is a $(2: 3)$-median semilattice.

Remark 3.3. As mentioned, conservative median semilattices $\mathbf{A}$ are (2:3)-median semilattices, whenever $|A| \geqslant 5$. Hence, Lemma 3 and Theorem 3 in [8] follow as corollaries of Theorem 3.2. In fact, the conservative median semilattice $\{0,1\}^{2}$ is the only "pathological" case.

\section{MEdiAn-HOMOMORPHiSMS OVER TREES}

In [8] the authors presented a study of median-homomorphims of the form

$$
f: \mathbf{A}_{1} \times \cdots \times \mathbf{A}_{n} \rightarrow \mathbf{B}_{1} \times \cdots \times \mathbf{B}_{k},
$$

in the case where the median algebras $\mathbf{A}_{1}, \ldots, \mathbf{A}_{n}$ and $\mathbf{B}_{1}, \cdots, \mathbf{B}_{k}$ are conservative. In the current paper we seek to generalise the results of [8] to arbitrary median algebras. To do so, we shall make use of the following general fact in universal algebra that we briefly recall; for further background see, e.g., [7, 11].

Let $\mathbf{A}_{1}, \cdots, \mathbf{A}_{n}$ and $\mathbf{B}_{1}, \cdots, \mathbf{B}_{k}$ be arbitrary algebras of the same type. For each $j \in[k]$, let $\pi_{j}$ denote the $j$-th projection on $\mathbf{B}_{1} \times \cdots \times \mathbf{B}_{k}$, i.e., the mapping

$$
\pi_{j}: \mathbf{B}_{1} \times \cdots \times \mathbf{B}_{k} \rightarrow \mathbf{B}_{j}
$$

Clearly, $\pi_{j}$ is a homomorphism. Also, for a function

$$
f: \mathbf{A}_{1} \times \cdots \times \mathbf{A}_{n} \rightarrow \mathbf{B}_{1} \times \cdots \times \mathbf{B}_{k},
$$

let $f_{j}, j \in[k]$, denote the composition

$$
f_{j}=\pi_{j} \circ f: \mathbf{A}_{1} \times \cdots \times \mathbf{A}_{n} \rightarrow \mathbf{B}_{j},
$$


so that $f=\left(f_{1}, \ldots, f_{k}\right)$. Then $f$ is a homomorphism if and only if every $f_{j}, j \in[k]$, is a homomorphism.

Hence, in the particular case when $\mathbf{A}_{1}, \cdots, \mathbf{A}_{n}$ and $\mathbf{B}_{1}, \cdots, \mathbf{B}_{k}$ are median algebras, the description of median-homomorphisms (4.1) is reduced to the description of median-homorphisms of the form

$$
f: \mathbf{A}_{1} \times \cdots \times \mathbf{A}_{n} \rightarrow \mathbf{B}
$$

from a finite product of median algebras $\mathbf{A}_{1}, \cdots, \mathbf{A}_{n}$ (thought of as median semilattices) to a median algebra $\mathbf{B}$. Now a general characterization of homomorphisms of the form (4.2) still eludes us, however we can provide explicit descriptions of such mappings when $\mathbf{B}$ is of a special type, namely, when $\mathbf{B}$ is a (2:3)-semilattice or, equivalently, when $\mathbf{B}$ is a tree.

Theorem 4.1. Let $\mathbf{A}_{1}, \cdots, \mathbf{A}_{n}$ be median algebras and let $\mathbf{B}$ be a tree. A mapping $f: \mathbf{A}_{1} \times \cdots \times \mathbf{A}_{n} \rightarrow \mathbf{B}$ is a median-homomorphism if and only if there is an $i \in[n]$ and a median-homomorphism $g: \mathbf{A}_{i} \rightarrow \mathbf{B}$ such that

$$
f\left(x_{1}, \ldots, x_{n}\right)=g\left(x_{i}\right), \quad \text { for all }\left(x_{1}, \ldots, x_{n}\right) \in A_{1} \times \cdots \times A_{n} .
$$

In other words, $f$ is an essentially unary median-homomorphism.

Proof. Clearly, sufficiency holds. To prove necessity we show that $f$ cannot depend on two different arguments. For the sake of a contradiction suppose that $f$ depends on at least 2 of its arguments. Without loss of generality, we may assume that they are the first two. Hence, there are $a, b \in \mathbf{A}_{1}$ and $\mathbf{c} \in \mathbf{A}_{1} \times \cdots \times \mathbf{A}_{n}$ such that $f\left(\mathbf{c}_{1}^{a}\right) \neq f\left(\mathbf{c}_{1}^{b}\right)$, where $\mathbf{c}_{i}^{x}$ denotes the tuple obtained from $\mathbf{c}$ by setting its $i$-th component to $x$.

Note that if $f\left(\mathbf{c}_{1}^{a}\right) \neq f\left(\mathbf{c}_{1}^{b}\right)$, then the same holds for all $\mathbf{d} \in \mathbf{A}_{1} \times \cdots \times \mathbf{A}_{n}$. Indeed, suppose to the contrary that there is $\mathbf{d} \in \mathbf{A}_{1} \times \cdots \times \mathbf{A}_{n}$ such that $f\left(\mathbf{d}_{1}^{a}\right)=f\left(\mathbf{d}_{1}^{b}\right)$. Then, from the fact that $f$ is median-preserving, it would follow that $f\left(\mathbf{c}_{1}^{a}\right)=f\left(\mathbf{c}_{1}^{b}\right)$ (a contradiction) since

$$
\begin{aligned}
\mathbf{m}\left(f\left(\mathbf{d}_{1}^{a}\right), f\left(\mathbf{c}_{1}^{a}\right), f\left(\mathbf{c}_{1}^{b}\right)\right) & =\mathbf{m}\left(f\left(\mathbf{d}_{1}^{b}\right), f\left(\mathbf{c}_{1}^{a}\right), f\left(\mathbf{c}_{1}^{b}\right)\right) \Leftrightarrow \\
f\left(\mathbf{m}\left(\mathbf{d}_{1}^{a}, \mathbf{c}_{1}^{a}, \mathbf{c}_{1}^{b}\right)\right) & =f\left(\mathbf{m}\left(\mathbf{d}_{1}^{b}, \mathbf{c}_{1}^{a}, \mathbf{c}_{1}^{b}\right)\right) \Leftrightarrow \\
f\left(\mathbf{c}_{1}^{a}\right) & =f\left(\mathbf{c}_{1}^{b}\right) .
\end{aligned}
$$

Hence, we must have $f\left(\mathbf{d}_{1}^{a}\right) \neq f\left(\mathbf{d}_{1}^{b}\right)$.

Similarly, if the second argument of $f$ is essential, then are $a^{\prime}, b^{\prime} \in \mathbf{A}_{2}$ and $\mathbf{c}^{\prime} \in \mathbf{A}_{1} \times \cdots \times \mathbf{A}_{n}$ such that $f\left(\mathbf{c}_{2}^{a^{\prime}}\right) \neq f\left(\mathbf{c}_{2}^{\prime{ }^{\prime}}\right)$. Once again, we have in fact that $f\left(\mathbf{d}_{2}^{\prime a^{\prime}}\right) \neq f\left(\mathbf{d}_{2}^{\prime b^{\prime}}\right)$, for all $\mathbf{d}^{\prime} \in \mathbf{A}_{1} \times \cdots \times \mathbf{A}_{n}$. Hence, we have that $f\left(\mathbf{c}_{1,2}^{x, y}\right)$ for $(x, y) \in\{a, b\} \times\left\{a^{\prime}, b^{\prime}\right\}$, are pairwise distinct. By picking $p$ of the form $f\left(\mathbf{c}_{1,2}^{x, y}\right)$, it then follows that the four points $f\left(\mathbf{c}_{1,2}^{x, y}\right) \in \mathbf{B}$ form a square as in Figure 4. This contradicts the fact that $\mathbf{B}$ is a tree.

By Theorem 4.1 to describe median-homomorphisms of the form

$$
f: \mathbf{A}_{1} \times \cdots \times \mathbf{A}_{n} \rightarrow \mathbf{B}
$$

in the case when $\mathbf{A}_{1}, \cdots, \mathbf{A}_{n}$ are median algebras and $\mathbf{B}$ is a tree, it suffices to describe median homomorphisms $f: \mathbf{A} \rightarrow \mathbf{B}$ for a median algebra $\mathbf{A}$ and a tree $\mathbf{B}$. Such descriptions follow from Theorem 2.2.

In the case when both $\mathbf{A}$ and $\mathbf{B}$ are trees, Theorem 2.2 together with Corollary 2.3 and Theorem 3.2, imply the following proposition.

Proposition 4.2. Suppose that the median algebras $\mathbf{A}$ and $\mathbf{B}$ are trees, and consider a mapping $f: A \rightarrow B$. Then the following assertions are equivalent.

(i) $f$ is a median-homomorphism. 


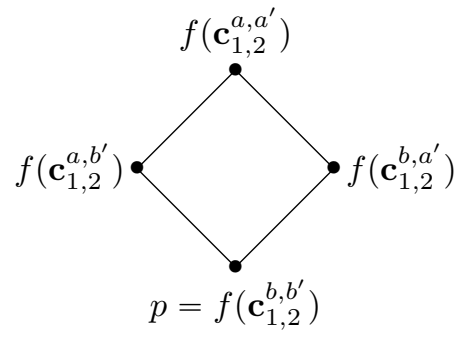

Figure 1. A square as in proof of Theorem 4.1.

(ii) For all $a, b \in A$, the induced mapping $f:\left\langle[a, b], \leqslant_{a}\right\rangle \rightarrow\left\langle[f(a), f(b)], \leqslant_{f(a)}\right\rangle$ is an isotone function between chains.

In the case when $\mathbf{A}$ is a tree and $\mathbf{B}$ is an arbitrary median algebra, Theorem 2.2 can be strengthened by an additional and equivalent assertion, namely, the existential variant of its assertion $(i i)$.

Theorem 4.3. Let $\mathbf{A}$ be a tree and $\mathbf{B}$ an arbitrary median algebra. Then the equivalent assertions in Theorem 2.2 are also equivalent to

$\left(i i^{\prime}\right)$ There is a $p \in A$ such that $f:\left\langle A, \wedge_{p}\right\rangle \rightarrow\left\langle B, \wedge_{f(p)}\right\rangle$ is a semilatticehomomorphism.

Proof. Obviously, $(i i) \Longrightarrow\left(i i^{\prime}\right)$. So to show that $\left(i i^{\prime}\right) \Longrightarrow(i)$, suppose that $\left\langle A, \wedge_{p}\right\rangle$ is a tree and that

$$
f:\left\langle A, \wedge_{p}\right\rangle \rightarrow\left\langle B, \wedge_{f(p)}\right\rangle
$$

is a semilattice-homomorphism. In particular, for every $a, b, c \in A$, we have that

$$
\mathbf{m}(a, b, c) \in\left\{a \wedge_{p} b, b \wedge_{p} c, c \wedge_{p} a\right\}
$$

and that $f:\left\langle A, \leqslant_{p}\right\rangle \rightarrow\left\langle B, \leqslant_{f(p)}\right\rangle$ is an order-homomorphism.

Without loss of generality, assume that $\mathbf{m}(a, b, c)=a \wedge_{p} b$. Hence,

$$
a \wedge_{p} b=\mathbf{m}(a, b, c)=\left(a \wedge_{p} b\right) \vee_{p}\left(b \wedge_{p} c\right) \vee_{p}\left(c \wedge_{p} a\right) \geqslant_{p} b \wedge_{p} c, c \wedge_{p} a,
$$

and, from the assumption that $f$ is a semilattice-homomorphism,

$f(\mathbf{m}(a, b, c))=f\left(a \wedge_{p} b\right)=f(a) \wedge_{f(p)} f(b) \geqslant_{f(p)} f(b) \wedge_{f(p)} f(c), f(c) \wedge_{f(p)} f(a)$.

Therefore,

$$
\begin{array}{ll}
\mathbf{m} & (f(a), f(b), f(c)) \\
= & \left(f(a) \wedge_{f(p)} f(b)\right) \vee_{f(p)}\left(f(b) \wedge_{f(p)} f(c)\right) \vee_{f(p)}\left(f(c) \wedge_{f(p)} f(a)\right) \\
= & f(a) \wedge_{f(p)} f(b)=f(\mathbf{m}(a, b, c))
\end{array}
$$

and the proof of $\left(i i^{\prime}\right) \Longrightarrow(i)$, and thus of the theorem, is now complete.

Remark 4.4. Note that the existential variant of ( iii) in Theorem 2.2 is not equivalent to $(i)$ even in the case when $\mathbf{A}$ is a tree. To see this, consider the function on $\mathbf{A}_{1}$ (see Figure 2(a)) that maps $a, b$ to $a$, and leaves $c$ and $d$ fixed. Then it is an order-homomorphism for $p=a$, but it is not a median-homorphism.

Remark 4.5. Note also that Theorem 4.3 does not necessarily hold in the case when $\mathbf{A}$ is not a tree, even if $\mathbf{B}$ is conservative. For instance, let $\mathbf{A}$ be the median algebra given in Figure 2(b) and $\mathbf{B}$ the 4-element chain 1,2,3,4, and consider the mapping that sends $d, d^{\prime}$ to $4, b, c$ to 2 , and $a$ to 1 . Then, for all $p \in A$, $f:\left\langle A, \wedge_{p}\right\rangle \rightarrow\left\langle B, \wedge_{f(p)}\right\rangle$ is an order-homomorphism, but it is not a semilatticehomorphism for $p=a$ (although it is a semilattice-homorphism for $p=d^{\prime}$ ). 


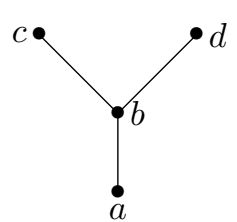

(a) $\mathbf{A}_{1}$

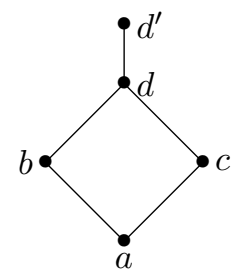

(b) $\mathbf{A}_{2}$

FigurE 2. Examples of $\wedge$-semilattices.

Problem. Given an arbitrary median algebra B, describe those median algebras A for which Theorem 4.3 holds.

Another natural question is to determine whether Theorem 4.1 still holds for a given median algebra $\mathbf{B}$. More precisely: Given arbitrary median algebras $\mathbf{A}_{1}, \cdots, \mathbf{A}_{n}$, describe those median algebras $\mathbf{B}$ for which all median-homomorphisms $f: \mathbf{A}_{1} \times$ $\cdots \times \mathbf{A}_{n} \rightarrow \mathbf{B}$ are essentially unary.

To answer this question, consider the following example. Suppose that $\mathbf{B}$ is an arbitrary median algebra thought as a $\wedge$-semilattice that is not a tree. Thus, there are $a, b \in B$ with a nontrivial upper bound $c$, i.e., $c>a, b>a \wedge b$. Set $p=a \vee b$, $q=a \wedge b$. Note that, together with $a$ and $b$, they form a square. Now, consider the median algebras $\mathbf{A}_{1}=\mathbf{A}_{2}=\{0,1\}$. Then $f: \mathbf{A}_{1} \times \mathbf{A}_{2} \rightarrow \mathbf{B}$ given by

$$
f(1,1)=p, \quad f(0,1)=a, \quad f(1,0)=b, \quad f(0,0)=q
$$

is a median homomorphism that depends on both of its variables.

Now this example can be easily extended to mappings $f: \mathbf{A}_{1} \times \cdots \times \mathbf{A}_{n} \rightarrow \mathbf{B}$ and, as we have seen, the condition that $\mathbf{B}$ is a $(2: 3)$-semilattice (i.e., a tree or, equivalently, does not contain a square as an order substructure) forces such median-preserving mappings to be essentially unary.

From these considerations, we have the following description of those median algebras $\mathbf{B}$ for which all median-homomorphisms $f: \mathbf{A}_{1} \times \cdots \times \mathbf{A}_{n} \rightarrow \mathbf{B}$ are trivial.

Corollary 4.6. All median-homomorphisms $f: \mathbf{A}_{1} \times \cdots \times \mathbf{A}_{n} \rightarrow \mathbf{B}$ are essentially unary if and only if $\mathbf{B}$ is a tree.

\section{REFERENCES}

[1] S. P. Avann. Metric ternary distributive semi-lattices. Proceedings of the American Mathematical Society, 12:407-414, 1961.

[2] H. J. Bandelt and J. Hedlíková. Median algebras. Discrete mathematics, 45:1-30, 1983.

[3] H.-J Bandelt, M. Van De Vel. The median stabilization degree of a median algebra. Journal of Algebraic Combinatorics, 9(2):115-127, 1999.

[4] J.-P. Barthélemy. Caractérisation médiane des arbres. Annals Discrete Math., 17:39-46, 1983.

[5] G. Birkhoff and S. A. Kiss. A ternary operation in distributive lattices. Bulletin of the American Mathematical Society, 53:749-752, 1947.

[6] G. Birkhoff. Lattice Theory, volume 25 of American Mathematical Society Colloquium Publications, revised edition. American Mathematical Society, New York, 1948.

[7] S. Burris, H. Sankappanavar. A course in universal algebra, GraduateTexts in Mathematics, Springer-Verlag, 1981.

[8] M. Couceiro, J.-L. Marichal and B. Teheux. Conservative median algebras and semilattices. Order 33(1): 121-132, 2016.

[9] B. A. Davey and H. A. Priestley. Introduction to lattices and order. Cambridge University Press, New York, second edition, 2002.

[10] E. Evans. Median lattices and convex subalgebras. In Universal Algebra, volume 29 of Colloquia Mathematica Societatis János Bolyai, pages 225-240. North-Holland, Amsterdam, 1982. 
[11] G. Grätzer. General lattice theory. Birkhäuser Verlag, Basel, second edition, 1998. New appendices by the author with B. A. Davey, R. Freese, B. Ganter, M. Greferath, P. Jipsen, H. A. Priestley, H. Rose, E. T. Schmidt, S. E. Schmidt, F. Wehrung and R. Wille.

[12] J. R. Isbell. Median algebra. Transactions of the American Mathematical Society, 260(2):319-362, 1980.

[13] M. Sholander. Trees, lattices, order, and betweenness. Proceedings of the American Mathematical Society, 3(3):369-381, 1952.

[14] M. Sholander. Medians, lattices, and trees. Proceedings of the American Mathematical Society, 5(5):808-812, 1954.

LORIA (CNRS - Inria Nancy Grand Est - Université de Lorraine), Équipe Orpailleur, Batiment B, Campus Scientifique, B.P. 239, F-54506 Vandoeuvre-lès-Nancy, France

E-mail address: miguel.couceiro[at]inria.fr

E-mail address: foldes.stephan[at]gmail.com

TEI of Epirus, PO Box 110, 47100 Arta, Greece

E-mail address: gmelet [at] teiep.gr 